\title{
Multidisciplinary design modeling and optimization for satellite with maneuver capability
}

\author{
Hai Huang • Haichao An • Wenrui Wu • \\ Lingyan Zhang • Beibei Wu - Weipeng Li
}

Received: 16 September 2013 / Revised: 23 February 2014 / Accepted: 15 March 2014 / Published online: 1 June 2014

(C) Springer-Verlag Berlin Heidelberg 2014

\begin{abstract}
According to the mission of a satellite with maneuver capability, the collaborative optimization (CO) method was introduced for the satellite system design, and the related multidisciplinary design optimization (MDO) model was established. The possessing and needed velocity increments $\Delta v$ and $\Delta v_{\text {need }}$ were taken as the measurement of maneuvering capability of the studied satellite, which were then combined with total mass of the satellite to form the optimization objective in the systematic level of the MDO problem. The design variables and constraints of the MDO problem dealt with disciplines or subsystems as guidance, navigation and control (GNC), power, and structure, and corresponding engineering analysis models were also built. A program system to solve the MDO problem was
\end{abstract}

Electronic supplementary material The online version of this article (doi:10.1007/s00158-014-1087-x) contains supplementary material, which is available toauthorized users.

H. Huang · H. An · W. Li $(\bowtie)$

Beihang University, Beijing 100191, China

e-mail: liweipeng@buaa.edu.cn

W. Wu

Institute of Manned Space System Engineering, China Academy of Space Technology, Beijing 100094, China

\section{Zhang}

Shanghai Institute of Satellite Engineering, Shanghai 200240, China

B. $\mathrm{Wu}$

Institute of Spacecraft System Engineering, China Academy of Space Technology, Beijing 100094, China developed by integrating a non-nested CO method, the commercial and user-supplied codes on framework software iSIGHT. The result showed that the satellite performance could be obviously improved, which also indicates MDO technique is feasible and effective for the spacecraft design problem. The modeling and optimization procedure of the work can be referred for further research and engineering design.

Keywords Multidisciplinary design optimization . Maneuver satellite $\cdot$ Collaborative optimization $\cdot$ Analysis models

\section{Introduction}

Satellite system design is a complex process involving lots of knowledge related to multiple disciplines, including space mission, payload, structure, orbit and attitude dynamics, power supply, thermal control and communication, etc. (Xu 2002). The designers usually need to tradeoff many essentials among them and it takes remarkable time in the procedure. How to efficiently organize the experts from different disciplinary groups and finally work out a satisfactory design is constantly concerned by spacecraft design departments.

Design problem can be transformed into an optimization problem in some cases. For instance, when entering a stage, the satellite system design can be stated as an optimization problem to determine a series of systematic parameters. However, such kind of a problem is extremely computational intensive, and inevitably requires multiple disciplinary analyses which are commonly conducted by different technical groups of a company, so that it is almost impossible to be solved by directly using methods for 
common mathematical programming problems. Although common optimization methods are widely introduced into some subsystem design problems, which usually only relate to individual disciplinary analyses for such as structure, orbit configuration, control respectively (Chen and Huang 2010; Sobieszczanski-Sobieski et al. 1985; Sobieszczanski-Sobieski and Haftka 1997)), the satellite systematic design and optimization problems are still hardly to be solved with the methods above.

For complex engineering optimization problems, multidisciplinary design optimization (MDO) was proposed by Sobieski (Sobieszczanski-Sobieski 1982; SobieszczanskiSobieski and Chopra 1990; Sobieszczanski-Sobieski 1990), which provided a layered approach to a complicated system optimization and seems especially suitable for system design problems with sub-systems. The method was originally developed for large-scale structural optimization problems combined with sub-structural analyses. Later, a lot of improved MDO algorithms (Cramer et al. 1994; Sobieszczanski-Sobieski 1989; Ilan et al. 1994; Braun 1996; Jaroslaw et al. 1998) were put forward, meanwhile several frame software like iSIGHT (EngineousSoftware 2004), ModelCenter (PhoenixIntegration 2008), ModeFrontier (Esteco 2011) and HyperStudy (Altair 2009) were developed. Now MDO are applied to many engineering system optimization fields, including aerospace and mechanical engineering (Renaud and Gabriele 1993; Brown and Olds 2006; Choi et al. 2006; Nobuhiro et al. 2005; Yokoyama et al. 2007; Blouin et al. 2004).

Spacecraft or satellite system design is a typical MDO problem which contains multiple subsystems and deals with different disciplinary analyses. Traditionally a spacecraft design is needed to collaborate with multiple groups in charge of corresponding sub-systems, in which discussions and compromises are often conducted. It is a time consuming process and can seldom reach a perfect result. By introducing $\mathrm{MDO}$ method into satellite system design, the efficiency of design can be expected to improve and then powerful technical supports can be obtained, which means better performance, faster design process and lower cost. It has been reported that MDO methods were successfully applied to a series of aerospace engineering programs and made sufficiently benefits through the design technology (Choi et al. 2006; Nobuhiro et al. 2005; Yokoyama et al. 2007; Blouin et al. 2004; Braun et al. 1997; Allison et al. 2006). These researches showed that MDO is effective and efficient in dealing with the coupling among multi-subsystems. The key problems to consider in engineering applications of MDO now lie in choosing the modeling technique for practical problems and implementing an optimization strategy.
Although MDO now becomes a very important technique in complex engineering design, most of the published journal papers only focused on theoretical methods of MDO. Some applications only simply gave final results or advantages after using MDO, even just mentioned as an event like advertising. However, as an engineering design technology, how to establish the MDO model of the practical object, which includes design variables, objective functions as well as considered constraints in system or subsystem level, is extremely significant. Meanwhile, the corresponding analysis models in multiple disciplines also must be determined. Commercial software like iSIGHT provides users a tool to form an MDO frame and implement the computational procedure. Even so, in the procedure users still have to develop or call additional codes to conduct model related computation or analysis. Therefore modeling is a key point for an engineering MDO problem.

According to the mission characteristics of a maneuver satellite, this paper discussed the measurement and analyzed the maneuvering capability of the studied satellite, i.e. the possessing and needed velocity increments $\Delta v$ and $\Delta v_{\text {need }}$, which were then combined with total mass of the satellite to form the optimization objectives in the systematic level of the MDO problem. Considering the deficiencies of $\mathrm{CO}$ (collaborative optimization) methods like convergence difficulties, an improved version of $\mathrm{CO}$ method, i.e. a non-nested CO (NNCO) method (Wu et al. 2012), was used for this problem. The design variables and constraints in the MDO problem dealt with disciplines or subsystems as GNC, Power, and Structure, and corresponding analysis models close to practical engineering situations were also established. A program system to solve the MDO problem was developed by integrating the NNCO method, the commercial and user-supplied codes on framework software iSIGHT. The result showed that the satellite performance considering both maneuvering capability and mass could be obviously improved with the program system, which also indicates the MDO technique is feasible and effective for spacecraft design problems. The modeling and optimization procedure of the work can be referred for further research works; however, more practical models for satellite design and analysis are still expected to develop.

The outline of this paper is organized as follows. Section 2 introduces concept of maneuver satellite and its velocity increment followed by system optimization modeling in Section 3. Section 4 presents the MDO modeling based on $\mathrm{CO}$ method and Section 5 details the disciplinary analysis modeling. The optimization results are presented in Section 6 and a brief conclusion is arrived at in Section 7. Additionally, more details in each disciplinary modeling as well as velocity increment calculations can be referred to the Electronic Supplementary Materials (ESM). 


\section{Maneuver satellite and its velocity increment of maneuver}

\subsection{Maneuver satellite and its missions}

The maneuver satellite studied in this paper is designed to serve other sun-synchronous satellites in the orbit height range of $400 \sim 1000 \mathrm{~km}$. It is initially launched into a parking orbit which usually is also one of sun-synchronous orbits. When needed, the designed satellite should be able to maneuver or transfer to the orbit of target satellite that requires service, and then rendezvous with the target satellite to provide orbital support. The maneuver process can be presented as in Fig. 1. That means the maneuver satellite must possess enough maneuver capability to reach any target satellite on the sun-synchronous orbit within given height range.

According to the definition and properties of sunsynchronous orbits, the inclination of sun-synchronous orbit changes with its height which follows the relation below:

$\cos i_{\text {sso }}=-0.09892\left(a / R_{e}\right)^{7 / 2}\left(1-e^{2}\right)^{2}$,

where $i_{s s o}$ and $a$ are the inclination and semi-major axis or radius of sun-synchronous orbit respectively, and $R_{e}$ is the radius of the Earth. From (1), it can be seen that the maneuver between sun-synchronous orbits, i.e. from parking orbit to target orbit, requires changing both height and inclination. Besides, it also needs to adjust the phase angle and the right ascension of ascending node when a maneuver satellite reaches a target satellite.

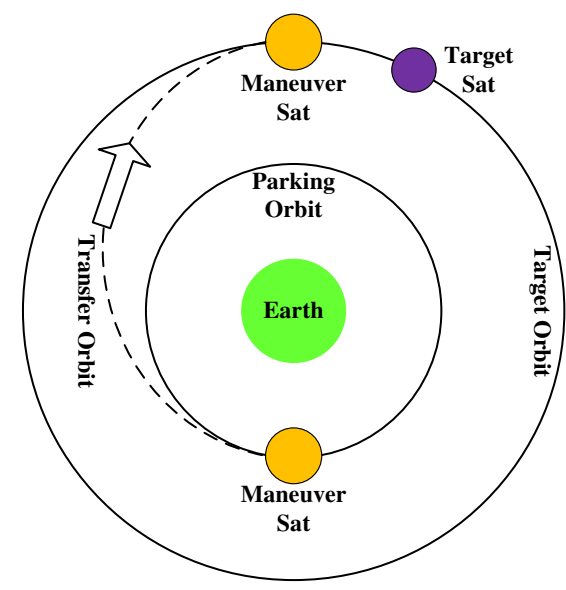

Fig. 1 Maneuver process of the satellite
2.2 Velocity increment for maneuver of orbital height and inclination

\subsubsection{Maneuver strategies}

There are many ways for the maneuver satellite to reach the target orbital height and inclination from its initial orbit. The frequently considered strategies are demonstrated as follow, where Strategies 1, 2, $3 \& 4$ are general approaches (Xiao 1995), and Strategy 4 achieved by analytical calculation. Based on these 4 common strategies, another maneuver strategy, Strategy 5, is proposed in this work as below.

Strategy 1 Firstly change the initial orbit to another circular orbit with the same height as the target orbit with two-impulse Hohmann maneuver law, then adjust the angle of inclination to that of target with 3rdvelocityimpulse.

Strategy 2 Firstly change the major semi-axis of the orbit to same as the target with a velocity impulse at ascending node (or descending node), then simultaneously round out the orbit and adjust its angle of inclination to that of target orbit with another single velocity impulse at descending node (or ascending node).

Strategy 3 At first simultaneously adjust the major semiaxis of the orbit and its angle of inclination to same as the target with a single velocity impulse at ascending node (or descending node), then round out the orbit with another velocity impulse at ascending node (or ascending node).

Strategy 4 First adjust the major semi-axis of the orbit to that of the target orbit and simultaneously change an angle $\delta$ of its inclination with a single velocity impulse at ascending node (or descending node), then round out the orbit and change the rest angle of its inclination to reach the target orbit with another velocity impulse at ascending node (or descending node), where angle $\delta$ is determined by minimizing total velocity increment $\Delta v_{a}$ for the maneuver.

Strategy 5 First adjust the major semi-axis of the orbit and simultaneously change one half of its inclination to reach that of the target orbit with a single velocity impulse at ascending node (or descending node), then round out the orbit and change the rest half of its inclination with another velocity impulse at ascending node(or ascending node). 
The total velocity increments $\Delta v_{\mathrm{a}}$ for the maneuver with above Strategies 1 to 5 are presented in Section 1 in the Electronic Supplementary Materials (ESM). In order to explain the superiority of proposed Strategy 5, comparisons among these strategies are made in Section 2.2.2.

\subsubsection{Comparison of strategies}

Figure 2 shows the vector relation for orbit maneuver, where $v_{c 1}$ and $v_{c 2}$ are the velocities of the initial (or parking) and final (or target) orbits respectively; $\Delta v_{1}$ and $\Delta v_{2}$ are the required velocity increments for changing the orbital major semi-axis (and/or rounding the orbit) and inclination respectively, while $\Delta v$ is the required velocity increment for simultaneously changing the orbital major semi-axis (and/or round the orbit) and inclination. It can be obviously found that $\Delta v<\Delta v_{1}+\Delta v_{2}$ from Fig. 2 .

Comparing strategy 1 and 2, it can be seen that the first impulse velocity increments of both strategies are equivalent, however, the second impulse of Strategy 2 in fact acts as the second and third impulses of the Strategy 1, i.e. simultaneously round the orbit and inclination. Therefore the velocity increment of Strategy 2 is less than that of Strategy 1.

On the other hand, it is well known that the higher the orbital velocity is, the more the velocity increment is needed for an inclination change. So Strategy 2 is better than Strategy 3 for maneuver from higher to lower altitudes, and on the contrary in reverse. Whether Strategy 5 is superior to Strategies 2 and 3 depends on the initial and final orbital altitudes to maneuver, however, it must be superior to one of them. Because the inclination angle change $\delta$ in its first impulse is optimized, Strategy 4 can reach the minimum velocity increment. Figure 3 shows the needed velocity increments to maneuver from the initial orbit (altitude range $400 \sim 900 \mathrm{~km}$ ) to final target orbit (altitude $1000 \mathrm{~km}$ ) with different 2-impulse strategies. In Fig. 3, the left ends and right of the curves actually just correspond to Strategies 2 and 3 respectively, and the middle parts with sign " $\nabla$ " correspond to Strategy 5 . It can be seen that Strategy 5 is very close to Strategy 4, i.e. the lowest point of each curve. For example, the top curve in Fig. 3 represents the required velocity increments to maneuver from 400 and $1000 \mathrm{~km}$ height of sun-synchronous orbits with the same

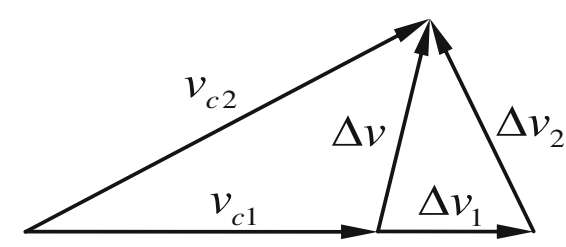

Fig. 2 Vector relation of orbit maneuver

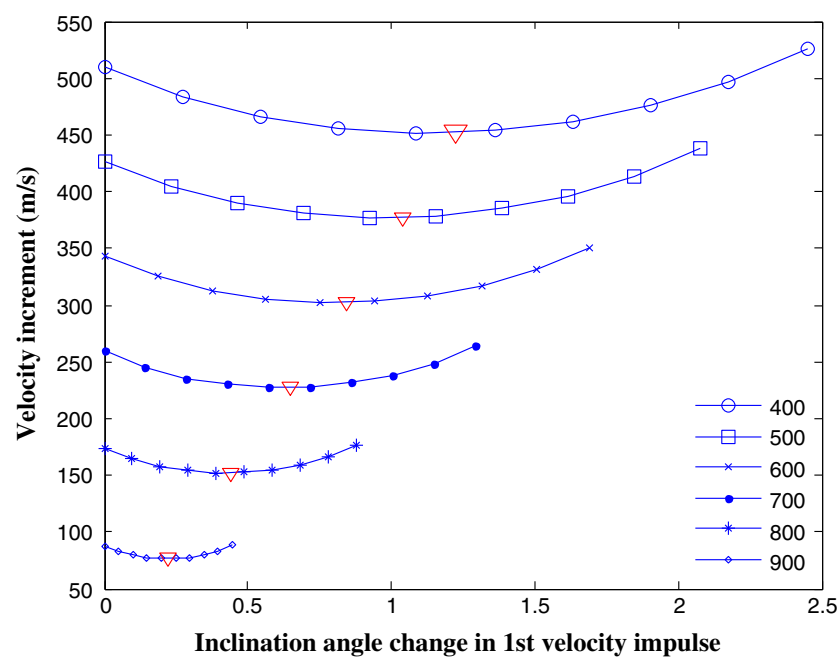

Fig. 3 Velocity increments to maneuver from orbit altitude range 400 $900 \mathrm{~km}$ to $1000 \mathrm{~km}$

ascending node $(\Delta \Omega=0)$, where the changes of orbital angle of inclination is $\Delta i=99.48-97.03=2.45^{\circ}$ according to the sun-synchronous orbital property. Table 1 shows the required velocity increments of aforementioned 5 strategies for the maneuvers between obits at heights $400 \mathrm{~km}$ and $1000 \mathrm{~km}$.

It can be seen from above study including Fig. 3 and Table 1 that Strategies 1 and 5 require maximum and minimum velocity increments respectively, and Strategy 5 is almost same as that of Strategy 4. As it is much simpler compared to Strategy 4, Strategy 5 is adopted to calculate the total maneuver velocity increment in this work.

2.3 Velocity increments to adjust orbital phase angle and right ascension of ascending node

After completing the maneuver of orbital height and inclination, there still could be difference in orbital phase angles and right ascensions of ascending nodes between maneuver satellite and target satellite, therefore it needs additional maneuver to adjust the differences.

\subsubsection{Maneuver to adjust orbital phase angle}

When the target satellite is leading the maneuver satellite with a phase angle $\theta$, the maneuver satellite needs to be applied a velocity increment $\Delta v_{\theta}$ in its moving opposite direction to reduce its speed and enter the phase adjust orbit, seeing in Fig. 4a. After flying around the Earth $n$ circles, the maneuver satellite catches up with the target satellite at the tangent point, then after applying another increment, it will enter the round target orbit again and complete the 
Table 1 Required velocity increments of 5 strategies

Maneuver from

height $(\mathrm{km})$

Strategy No.

\begin{tabular}{llllll} 
& 1 & 2 & 3 & 4 & 5 \\
\hline 400 to 1000 & 632.48 & 509.25 & 525.66 & 451.69 & 452.10 \\
1000 to 400 & 632.48 & 525.66 & 509.25 & 451.69 & 451.69 \\
\hline
\end{tabular}

adjust of phase. According to orbital dynamics, we give the total velocity increment of this procedure as below

$\Delta v_{\theta}=2 \sqrt{\frac{\mu}{R}} \cdot\left[1-\sqrt{1-\frac{\Delta h}{2 R-\Delta h}}\right]$,

$\Delta h=2 R \cdot\left[1-\sqrt[3]{\left(1-\frac{\Delta \theta}{360}\right)^{2}}\right]$,

where $R$ is the radius of target orbit, $\Delta \theta=\theta / n$ is the adjusted phase angle in degree per circle, and the catching circle number $n$ can be determined by following relation

$n\left|T-T_{p}\right|=\frac{\theta}{360} \cdot T$,

where $T$ and $T_{p}$ are the periods of target and phase adjust orbits respectively.

In the case that the maneuver satellite is lagging the target satellite with a phase angle $\theta$ as in Fig. 4b, a similar maneuver is required to conduct, and the velocity increment can be given as follows in (5) and (6), while their directions are opposite to that of the leading phase case.

$$
\begin{aligned}
& \Delta v_{\theta}=2 \sqrt{\frac{\mu}{R}} \cdot\left[\sqrt{1+\frac{\Delta h}{2 R+\Delta h}}-1\right], \\
& \Delta h=2 R \cdot\left[\sqrt[3]{\left(1+\frac{\Delta \theta}{360}\right)^{2}}-1\right] .
\end{aligned}
$$

\subsubsection{Maneuver to change orbital right ascension of ascending node}

Because of very large velocity increment needed, orbital right ascensions of ascending node almost cannot be remarkably adjusted. The velocity increment for small right ascensions of ascending node change can be stated as (Xiao 1995)

$\Delta v_{h}=\frac{\sqrt{\mu p} \sin i}{r \sin u} \Delta \Omega$

where $\Delta \Omega$ is the change of right ascension of ascending node, and $\Delta v_{h}$ is the corresponding velocity increment. Figure 5 shows the change of needed velocity increment vs. local time past descending node and height of sunsynchronous orbit, where the change of local time exactly corresponds to $\Delta \Omega$, i.e. $15 \mathrm{deg}$ per hour.

From Fig. 5 it can be easily found that velocity increment increases much more quickly with the change of local time than that of orbital height.

\section{System optimization modeling of maneuver satellite}

To conduct optimization of maneuver satellite, first of all it is needed to establish the model of the optimization problem, which includes the objective function, design variables and constraints of the problem.
Fig. 4 Maneuver to adjust phase angle. a Leading phase adjusting. b Lagging phase adjusting

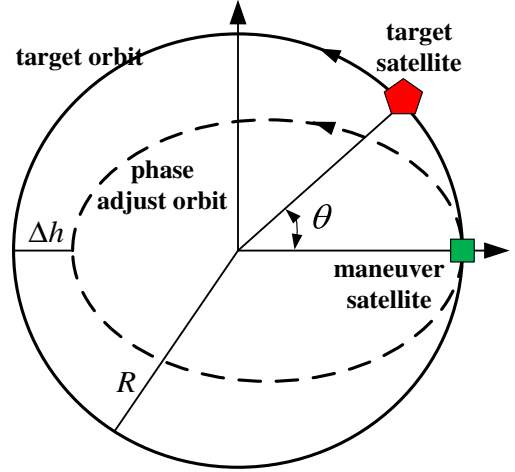

(a)

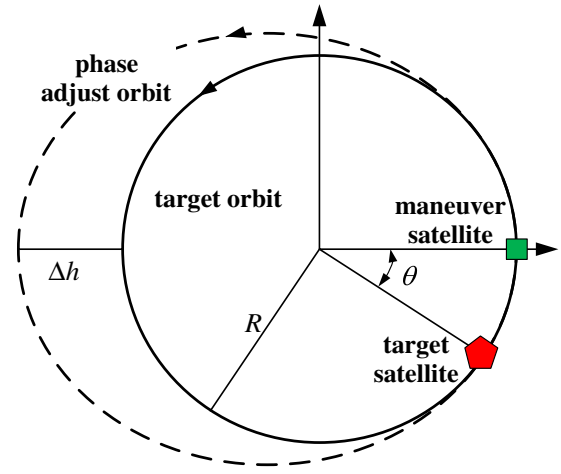

(b) 


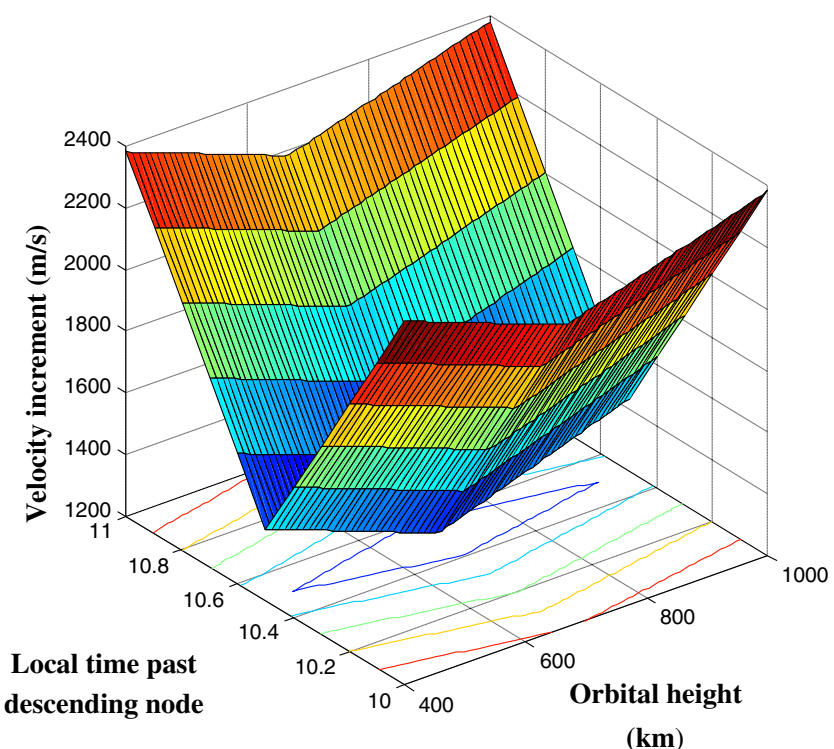

Fig. 5 Velocity increment vs. local time past descending node and orbital height

\subsection{Objective function of system optimization}

As the maneuver capability measured by velocity increment is the most important for a maneuver satellite, meanwhile the total mass of satellite is always expected to be as low as possible, and the optimization objective of this work is taken as

$\max \frac{\Delta v-\Delta v_{\text {need }}}{m_{\text {total }}}$,

where $m_{\text {total }}$ is the satellite mass; $\Delta v$ is the velocity increment the satellite possesses, and $\Delta v_{\text {need }}$ is the velocity increment the satellite needs to realize the maneuver mission, which can be presented as

$\Delta v_{\text {need }}=\Delta v_{a}+\Delta v_{\theta}+\Delta v_{\Omega}$, where $\Delta v_{a}, \Delta v_{\theta}$ and $\Delta v_{\Omega}$ are needed increments for maneuvers of height \& inclination, phase angle and the right ascension of ascending node respectively.

Equation (8) can be written as a non-dimensional form

$\max f(X)=\frac{\left(\Delta v-\Delta v_{\text {need }}\right) / \Delta v_{\text {scale }}}{m_{\text {total }} / m_{\text {total }}^{\text {sal }}}$,

where $f(X)$ is the objective function; $\Delta v_{\text {scale }}$ and $m_{\text {total }}^{\text {scale }}$ are parameters for non-dimensional process.

\subsection{Design variables and constraints of optimization problem}

The design variables and constraints of this work deal with system and orbital performances, subsystems of attitude control, power and structure. The design variables are shown in Table 2.

The constraints considered in the problem are shown in Table 3.

\section{MDO modeling based on $\mathrm{CO}$ method}

The established optimization model in the section above usually is a very complicated multidisciplinary design optimization (MDO) problem which can be hardly solved by common method. Collaborative optimization (CO), a decomposition-based MDO method, is generally used in aeronautical systems (Ilan et al. 1994; Yokoyama et al. 2007). However, the subsystems optimizations of CO method are nested into system optimization of it in the execution process, numerous subsystem analyses are required to achieve consistency for entire system (Alexandrov and Lewis 2002). For this problem, a non-nested collaborative optimization (NNCO) method was developed in our

Table 2 Design variables chosen for optimization problem

\begin{tabular}{|c|c|c|c|c|}
\hline Classifications & No. & Designations & Symbols & Ranges \\
\hline \multirow[t]{3}{*}{ System \& orbit } & \multirow[t]{3}{*}{3} & Velocity increment $(\mathrm{m} / \mathrm{s})$ & $\Delta v$ & {$[1000,3000]$} \\
\hline & & Orbital height $(\mathrm{km})$ & $h$ & {$[400,1000]$} \\
\hline & & Local time past descending node & DNT & {$[10,11]^{\mathrm{a}}$} \\
\hline Attitude control & 1 & Angular momentum of reaction wheel (Nms) & $H_{w h e e l}$ & {$[0.5,15]$} \\
\hline \multirow[t]{2}{*}{ Power } & \multirow[t]{2}{*}{2} & Area of solar array $\left(\mathrm{m}^{2}\right)$ & $A_{s a}$ & {$[5,10]$} \\
\hline & & Battery capacity (Ah) & $C_{s}$ & {$[30,100]$} \\
\hline Structure & 24 & Sizes of components & $X_{s t r}$ & Table 9 \\
\hline
\end{tabular}

${ }^{a}$ considering the large amount of velocity increment required to change local time past descending node, $D N T$ is taken as constant in this paper, i.e. $D N T=10.5$ 
Table 3 Constraints considered in optimization problem

\begin{tabular}{|c|c|c|c|}
\hline Classifications & No. & Designations & Limitations \\
\hline \multirow[t]{4}{*}{ System \& orbit } & \multirow[t]{4}{*}{4} & Residue of velocity increment $(\mathrm{m} / \mathrm{s}) \Delta v-\Delta v_{\text {need }}$ & $\geq 0$ \\
\hline & & Orbital height $(\mathrm{km}) h^{s}$ & {$[400,1000]$} \\
\hline & & Total mass of satellite $(\mathrm{kg}) m_{\text {total }}$ & $\leq 1250$ \\
\hline & & Propellant mass $(\mathrm{kg}) m_{p}$ & $\leq 600$ \\
\hline Attitude control & 1 & Residue of Angular momentum (Nms) $g_{G N C}$ & $\geq$ \\
\hline \multirow[t]{3}{*}{ Power } & \multirow[t]{3}{*}{3} & Power residue (W) $P_{\text {saEOL }}-(1+5 \%) P_{S C}$ & $\geq$ \\
\hline & & Battery average discharge depth $\mathrm{DOD}_{\text {Avg }}$ & $30 \%$ \\
\hline & & Battery maximum discharge depth $\mathrm{DOD}_{\max }$ & $35 \%$ \\
\hline Structure & 1 & 1st natural frequency $(\mathrm{Hz}) f_{1}$ & 22 \\
\hline
\end{tabular}

The meanings of some parameters such as $g_{G N C}, P_{S a E O L}$ and $P_{S C}$ will be explained in later modeling of disciplinary optimizations

previous work (Wu et al. 2012) and introduced for the MDO problem in this work. With this $\mathrm{CO}$ method, the original system optimization problem was transformed into a system level optimization problem and $N$ disciplinary optimization problems (see Fig. 6). In this work, besides system level optimization that aims at the system and orbital object and requirements of the satellite, the disciplinary optimizations mainly consider the requirements of the attitude control, power and structural subsystems respectively which are all solved by sequential quadratic programming (SQP) methods. No matter how system level optimization problem or disciplinary optimization problems, they were taken as temporarily decoupled each other. For example, in solving system level optimization problem, only system design variables $x^{s}=\left\{x_{1}^{s}, \cdots, x_{N}^{s}\right\}$ and coupled variables $y^{s}=\left\{y_{1}^{s}, \cdots, y_{N}^{s}\right\}$ could be changed and other parameters like $x^{*}$ and $y^{*}$ remained constant. Similarly, in solving the $j$-th disciplinary optimization problem, only $x_{j}$ and $y_{j}$ could be changed. Once the iterations of $\mathrm{CO}$ method come to convergence, the system variables will get close to disciplinary variables, i.e. $J_{j}^{s}=0, J_{j}=0(j=1, \cdots, N)$. By using this kind of treatment, this optimization strategy proved to be effective and more efficient based on the results of analytical and numerical examples in (Wu et al. 2012).

\subsection{Model of system level optimization}

The system level optimization model of the maneuver satellite MDO problem can be stated as below

$$
\begin{array}{ll}
\text { Find } & X_{\text {sys }}=\left\{h^{s}, A_{\text {sa }}^{s}, \Delta v, m_{G N C}^{s}, P_{G N C}^{s}, m_{\text {Power }}^{s}, m_{\text {str }}^{s}\right\}^{T} \\
\text { Max } & P\left(X_{\text {sys }}\right)=\frac{\left(\Delta v-\Delta v_{\text {need }}\right) / \Delta v_{\text {scale }}}{m_{\text {total }} / m_{\text {total }}^{\text {scal }}} \\
\text { s.t. } & J_{G N C}^{s} \leq 10^{-6} \\
& J_{\text {Power }}^{s} \leq 10^{-6} \\
& J_{\text {Str }}^{s} \leq 10^{-6} \\
& \Delta v_{\text {need }} \leq \Delta v \\
& m_{p} \leq 500 \\
& m_{\text {total }} \leq 1500 \\
& X_{\text {sys }}^{L B} \leq X_{\text {sys }} \leq X_{\text {sys }}^{U B}
\end{array}
$$

where $X_{\text {sys }}, X_{\text {sys }}^{L B}$ and $X_{\text {sys }}^{U B}$ are system design variable vector and its lower and upper bonds; $h^{s}$ is the orbital altitude, $A_{s a}^{s}$ is the area of solar array, $m_{G N C}^{s}$ and $P_{G N C}^{s}$ are the mass and power consume of control subsystem respectively,
Fig. 6 Principle block diagram of $\mathrm{CO}$ method

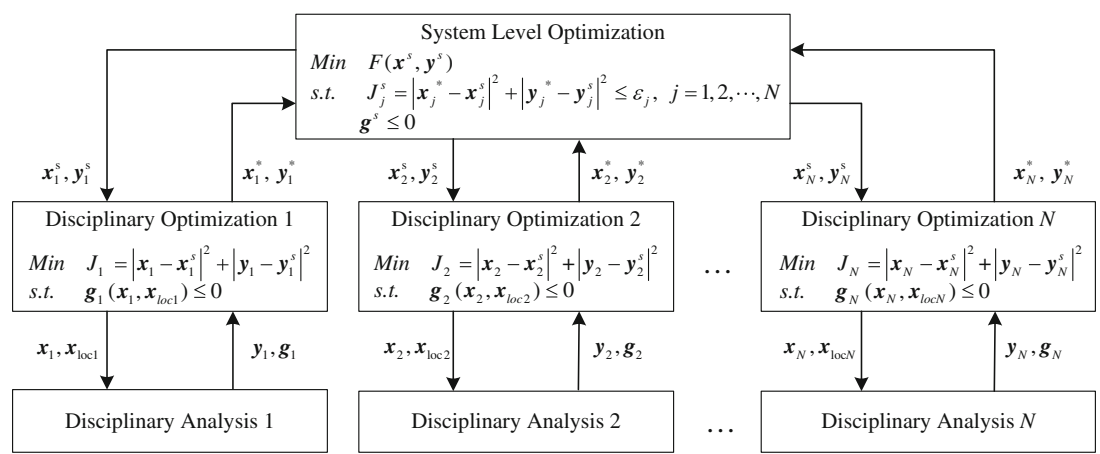


Fig. 7 Optimization framework

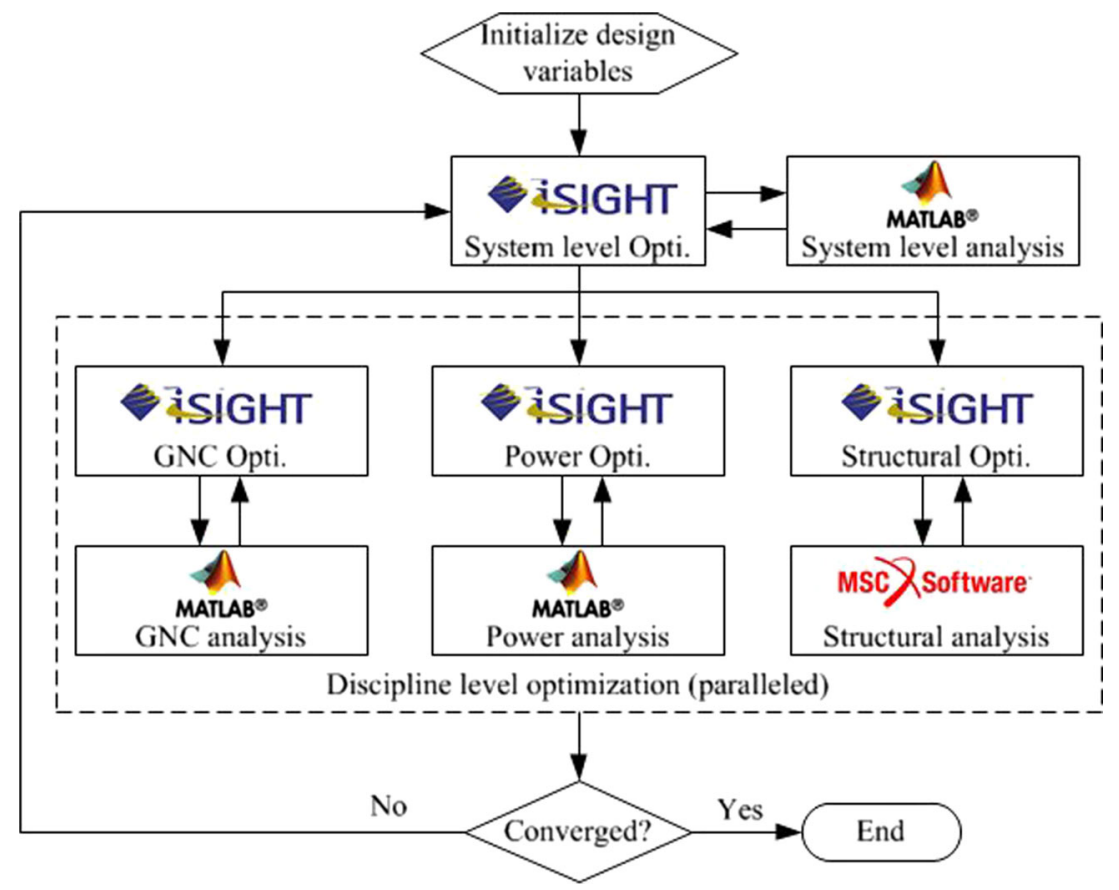

$m_{\text {Power }}^{s}$ and $m_{\text {str }}^{s}$ are masses of power and structure subsystems respectively; $J_{G N C}^{S}, J_{P o w e r}^{S}$ and $J_{S t r}^{S}$ are compatibility constraint functions for control, power and structure disciplinary respectively, which are presented as same as the functions $J_{G N C}, J_{\text {Power }}$ and $J_{S t r}$ in disciplinary optimization problems, and superscript " $s$ " here expresses the corresponding variables or parameters which are changeable in system level optimization.

For solving the problem more efficiently and avoiding the difficulty from compatibility constraints, in practical computation the problem (11) was rewritten as the following form in which the compatibility constraints were substituted by exterior point penalty terms of the objective function (Tan et al. 2008) in (12),

$$
\begin{gathered}
\text { Find } X_{\text {sys }}=\left\{h^{s}, A_{s a}^{s}, \Delta V, m_{G N C}^{s}, P_{G N C}^{s}, m_{\text {Power }}^{s}, m_{\text {str }}^{s}\right\}^{T} \\
\text { Min } F\left(X_{\text {sys }}\right)=- \\
\begin{array}{c}
W \cdot \frac{\left(\Delta v-\Delta v_{\text {need }}\right) / \Delta v_{\text {scale }}}{m_{\text {total }} / m_{\text {total }}^{\text {scal }}} \\
+\gamma \cdot \sum_{i=1}^{3} \max \left(J_{i}^{s}-10^{-6}, 0\right)
\end{array}
\end{gathered}
$$

s.t. $\quad \Delta v_{\text {need }} \leq \Delta v$

$$
\begin{aligned}
& m_{p} \leq 500 \\
& m_{\text {total }} \leq 1500 \\
& X_{\text {sys }}^{L B} \leq X_{\text {sys }} \leq X_{\text {sys }}^{U B}
\end{aligned}
$$

Table 4 Conditions to calculate disturbance moments

\begin{tabular}{ll}
\hline Designations & Parameters \\
\hline Orbit type & Sun-synchronous \\
Height & $850 \mathrm{~km}$ \\
Local time past descending node & $10: 30 \mathrm{am}$ \\
Principal moments of inertia $\left\{I_{x x}, I_{y y}, I_{z z}\right\}^{T}$ & $\{375,465,480\}^{T} \mathrm{kgm}^{2}$ \\
Solar panel Area, as the sunlit surface & $5.5 \mathrm{~m}^{2}$ \\
Radius vector of solar pressure center $\mathbf{l}_{p}$ & $\{0.36,0,0.36\}^{T} \mathrm{~m}$ \\
Radius vector of aerodynamic force center $\mathbf{l}$ & $\{0.36,0.36,0.36\}^{T} \mathrm{~m}$ \\
Internal magnetic torque $\mathbf{M}$ & $\{1.0,1.0,1.0\}^{T}$ Am \\
Face area $A_{P}$ & $8.38 \mathrm{~m}^{2}$ \\
Roll angle $\varphi$ & 10 \\
Pitch angle $\theta$ & $1{ }^{\circ}$ \\
Starting time of simulation & $0: 00$ of the Summer Solstice day, 2008
\end{tabular}


Fig. 8 Practical disturbance moment and its approximation
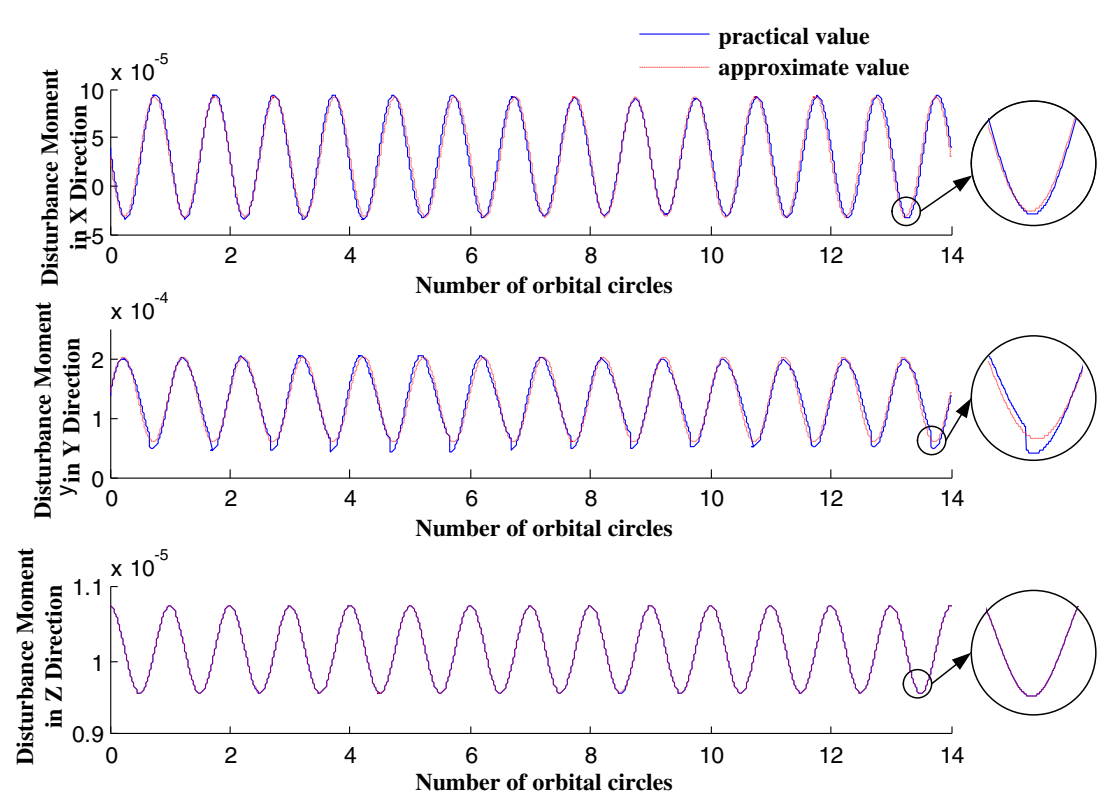

where $W$ was a weighted coefficient, and $\gamma$ was the penalty parameter that became larger as iteration increases.

\subsection{Model of control disciplinary optimization}

The model of control disciplinary optimization was stated as below in the paper,

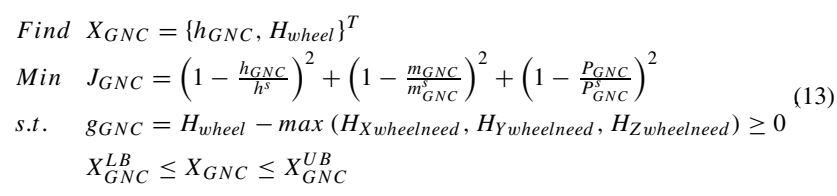

where $X_{G N C}, X_{G N C}^{\mathrm{LB}}$ and $X_{G N C}^{\mathrm{UB}}$ are the design variable vector of the control disciplinary as well as its lower and upper bonds; $h_{G N C}$ is the orbital altitude, $H_{w h e e l}$ is the angular momentum capacity of reaction wheel; $H_{X \text { wheelneed }}, H_{Y \text { wheelneed }}$ and $H_{Z \text { wheelneed }}$ are the components of needed angular momentum in $X, Y$ and $Z$ directions of body-fixed system whose coordinate axes just take the principal axes of inertia, $m_{G N C}$ and $P_{G N C}$ are the mass and consuming power of control subsystem respectively. The variables or parameters with subscript "GNC" but without superscript " $s$ " here may have the same physical meanings as the corresponding parameters with superscript " $s$ ", however they are changeable in the control disciplinary optimization. For example, both $m_{G N C}$ and $m_{G N C}^{s}$ denote the mass of control subsystem, but $m_{G N C}$ varies and $m_{G N C}^{s}$ keeps constant in optimization model (13). Similar sign usage also appears in later disciplinary optimization models.

\subsection{Model of power disciplinary optimization}

The model of power disciplinary optimization was stated as below,

$$
\begin{array}{ll}
\text { Find } & X_{\text {Power }}=\left\{h_{\text {Power }}, A_{s a}, C_{s}\right\}^{T} \\
\text { Min } & J_{\text {Power }}=\left(1-\frac{h_{\text {Power }}}{h^{s}}\right)^{2}+\left(1-\frac{A_{s a}}{A_{s a}^{s}}\right)^{2}+\left(1-\frac{m_{\text {Power }}}{m_{\text {Power }}^{s}}\right)^{2} \\
\text { s.t. } & P_{\text {SaEOL }}-(1+5 \%) P_{S C} \geq 0 \\
& \text { DOD } D_{\text {Avg }} \leq 30 \% \\
& \text { DOD } D_{\text {max }} \leq 35 \% \\
& X_{\text {Power }}^{L B} \leq X_{\text {Power }} \leq X_{\text {Power }}^{U B}
\end{array}
$$

where $X_{\text {Power }}, X_{\text {Power }}^{\mathrm{LB}}$ and $X_{\text {Power }}^{\mathrm{UB}}$ are the design variable vector of the power disciplinary as well as its lower

Table 5 Furrier coefficients and 1-circle accumulation components

\begin{tabular}{llrrr}
\hline $\mathrm{i}$ & $\mathrm{a}_{i 0}$ & \multicolumn{1}{c}{$\mathrm{a}_{i 1}$} & \multicolumn{1}{c}{$\mathrm{b}_{i 1}$} & \multicolumn{1}{c}{$\Delta H_{i}^{I}$} \\
\hline$X$ & $3.01846 \mathrm{E}-05$ & $-6.58269 \mathrm{E}-07$ & $-6.22857 \mathrm{E}-05$ & $9.23010 \mathrm{E}-02$ \\
$Y$ & $1.30772 \mathrm{E}-04$ & $1.22020 \mathrm{E}-05$ & $7.08999 \mathrm{E}-05$ & $7.99773 \mathrm{E}-01$ \\
$Z$ & $1.01542 \mathrm{E}-05$ & $5.96276 \mathrm{E}-07$ & $1.45241 \mathrm{E}-11$ & $-1.88639 \mathrm{E}-01$ \\
\hline
\end{tabular}


Table 6 Working mode arrangement during a day

\begin{tabular}{|c|c|c|c|c|c|}
\hline Circle No. & Working modes & Circle No. & Working modes & Circle No. & Working modes \\
\hline 1 & A & 6 & A & 11 & B \\
\hline 2 & $\mathrm{C}$ & 7 & A & 12 & $\mathrm{~B}$ \\
\hline 3 & A & 8 & A & 13 & A \\
\hline 4 & A & 9 & A & 14 & A \\
\hline 5 & A & 10 & $\mathrm{~B}$ & & \\
\hline
\end{tabular}

and upper bonds; $h_{\text {Power }}$ is the orbital altitude, $A_{s a}$ and $C_{S}$ are the solar array area and the battery capacity of the power subsystem, $P_{S a E O L}$ is the power that the solar array can supply in end of lifetime, $P_{S C}$ is the required lower bound of solar array output power, $D O D_{A v g}$ and $D O D_{\max }$ are the battery average and maximum discharging depths. The variables or parameters with subscript "Power" or " $s a$ " but without superscript " $s$ " here are changeable in the power disciplinary optimization.

\subsection{Model of structural disciplinary optimization}

The model of structural disciplinary optimization was stated as below,

$$
\begin{array}{ll}
\text { Find } & X_{\text {Str }}=\left\{W_{\text {Beam }}, H_{\text {Beam }}, T_{\text {Shell }}\right\}^{T} \\
\text { Min } & J_{\text {Str }}=\left(1-\frac{m_{\text {Str }}}{m_{\text {Str }}^{s}}\right)^{2} \\
\text { s.t. } & g_{\text {Str }}=f \geq 22_{1} \\
& X_{\text {Str }}^{L B} \leq X_{\text {Str }} \leq X_{\text {Str }}^{U B}
\end{array}
$$

where $X_{S t r}, X_{S t r}^{\mathrm{LB}}$ and $X_{\text {Str }}^{U B}$ are the design variable vector of the structural disciplinary as well as its lower and upper bonds; $W_{\text {Beam }}$ and $H_{\text {Beam }}$ are cross-sectional widths and depths of beam frame respectively; $T_{\text {Shell }}$ is equivalent thicknesses of panels; $m_{S t r}^{\mathrm{s}}$ is the structural mass and changeable in structural disciplinary optimization. $W_{\text {Beam }}, H_{\text {Beam }}$ and $T_{\text {Shell }}$ are all 8 component sub-vectors, therefore the number of total design variables in model (15) is 24 .

\subsection{System optimization framework}

Based on the system and disciplinary systems, the optimization problem is set up in iSIGHT, while analysis model of GNC and power disciplines are established in MATLAB, and structure discipline analysis model is implemented in MSC.Patran/Nastran. It is worthy to notice that the sensitivities Nastran output are that of design variables to satellite total mass. To transform them to the sensitivities of design variables to compatibility function, use the following formula:

$\frac{d J_{s t r}}{d X}=-\frac{2 \sqrt{J_{s t r}}}{m_{s t r}^{s}} \frac{d m_{s t r}}{d X}$.

The flow chart of the system optimization framework is shown in Fig. 7. Little different from normal CO method, the execution order has been modified with NNCO. Discipline level optimization is executed after system level
Fig. 9 FE models (some elements are erased). a model of whole satellite. $\mathbf{b}$ model of beam frame

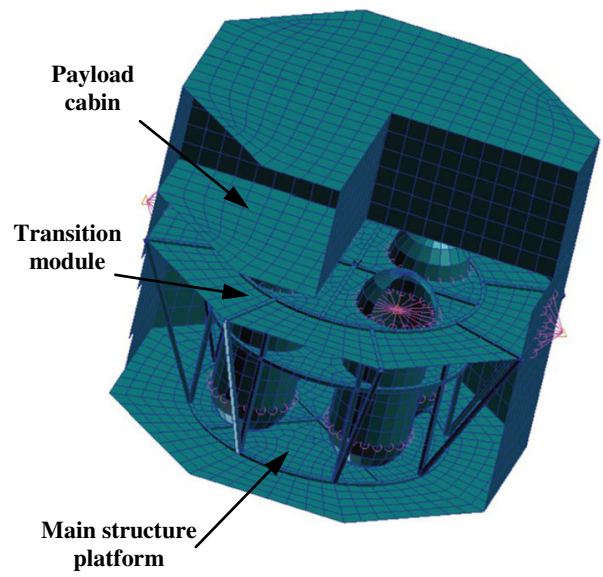

(a)

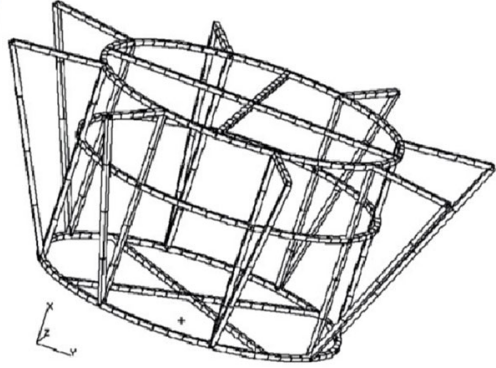

(b) 


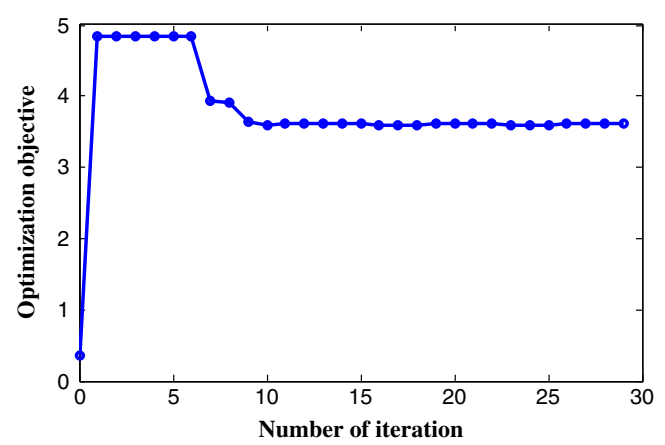

Fig. 10 Iteration history of objective function

optimization. In other words, the values of coupled parameters from disciplines design are fixed in system level optimization, and the values of coupled parameters from system design are fixed either in discipline level optimizations while the values are transferred after system or discipline level optimization. The executions of iSIGHT in the workstations are triggered by the completion of receiving the inputs data file. The completion flag of optimization execution is a file generated by iSIGHT with a user defined tool command language block.

\section{Disciplinary analysis modeling}

The established MDO models of satellite (12) (14) consequentially contain responding functions or state parameters such as the needed angular momentum components in $X, Y$ and $Z$ directions of body-fixed coordinate system $H_{X \text { wheelneed }}, H_{Y \text { wheelneed }}, H_{Z \text { wheelneed }}$ and the control subsystem mass $m_{G N C}$ in (13), which have to be solved through system or disciplinary level analysis. The system level optimization model mainly deals with the velocity increment $\Delta v_{\text {need }}$ needed to maneuver, the calculation of which was presented in Section 2 of the paper. The section below describes the analysis modeling for other responding functions or state parameters in MDO model.

\subsection{Analysis modeling for control disciplinary}

Control disciplinary optimization needs the analysis models to compute responding functions $H_{X \text { wheelneed }}, H_{Y}$ wheelneed,

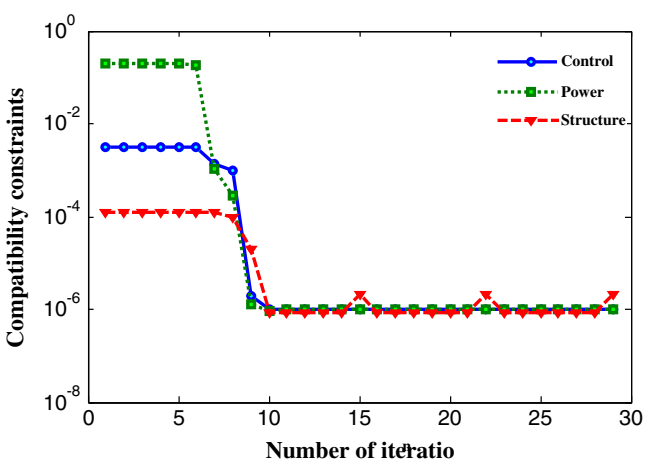

Fig. 11 Iteration history of compatibility constraints

$H_{Z \text { wheelneed }}, m_{G N C}$ and $P_{G N C}$, which are the needed angular momentums of reaction wheels as well as the mass and power of control subsystem. The needed angular momentums of the reaction wheels here are designed, during a required unloading period, to be able to counteract, the disturbance moment impulses resulting from the gravity gradient, aerodynamic force, solar pressure and Earth magnetic field. Then mass and power are determined according to the relations between momentum and them.

\subsubsection{Calculation of disturbance moments}

In order to find out angular momentum needed, formulas in reference (Wertz and Larson 1992) are used to calculate the outside disturbance moments from gravity gradient, the aerodynamic force, the solar pressure, and the Earth magnetic moment. In control subsystem design, the combined effect of disturbance moments is usually presented as the following approximate model by using Fourier expansions and only the 1st order harmonic wave terms remained and they are summed up and projected in body-fixed coordinate.

$$
\begin{aligned}
& T_{e x}=a_{x 0}+a_{x 1} \cos \omega_{0} t+b_{x 1} \sin \omega_{0} t \\
& T_{e y}=a_{y 0}+a_{y 1} \cos \omega_{0} t+b_{y 1} \sin \omega_{0} t, \\
& T_{e z}=a_{z 0}+a_{z 1} \cos \omega_{0} t+b_{z 1} \sin \omega_{0} t
\end{aligned}
$$

where $a_{i j}, b_{i 1}(i=x, y, z j=0,1)$ are Fourier coefficients obtained by using accurate model detailed in the ESM of Section 2.

Table 7 Optimization results of objectives

\begin{tabular}{llllr}
\hline Designations & Symbols & Initial values & Optimization results & Relative changes \\
\hline Velocity increment $(\mathrm{m} / \mathrm{s})$ & $\Delta v$ & 1350 & 1407.5564 & $4.26 \%$ \\
Velocity increment $(\mathrm{m} / \mathrm{s})$ & $\Delta v_{\text {need }}$ & 1331.6283 & 1219.67 & $-8.41 \%$ \\
Total mass of satellite $(\mathrm{kg})$ & $m_{\text {total }}$ & 1278.50 & 1250.4 & $-2.20 \%$ \\
Optimization objective & $P(Z)$ & 0.1410 & 3.6063 & $2456.98 \%$ \\
\hline
\end{tabular}


Table 8 Optimization results of global design variables

\begin{tabular}{lllllll}
\hline Classifications & No. & Designations & Lower bounds & Upper bounds & Initial values & Optimization results \\
\hline System & 1 & Velocity increment $(\mathrm{m} / \mathrm{s})$ & 1000 & 3000 & 1350 & 1407.5564 \\
Orbit & 1 & Orbital height $(\mathrm{km})$ & 400 & 1000 & 850 & 684.73 \\
Attitude control & 1 & Angular momentum of reaction wheel (Nms) & 0.5 & 15 & 4 & 3.03 \\
Power & 2 & Area of solar array $\left(\mathrm{m}^{2}\right)$ & 5 & 10 & 6 & 5.49 \\
& & Battery capacity $(\mathrm{Ah})$ & 30 & 100 & 40 & 34.93 \\
\hline
\end{tabular}

\subsubsection{Calculation of responding function}

Ignoring the slow procession of the orbital plane and considering the altitude angles $\varphi$ and $\theta$ to be very small, the 1-circle accumulation components of the disturbance moment impulse in $X, Y$ and $Z$ of an inertial coordinate system, in which $Y$ axis is perpendicular to orbital plane, can be determined as

$$
\begin{aligned}
& \Delta H_{X}^{I}=\frac{T}{2}\left(a_{x 1}-b_{z 1}\right) \\
& \Delta H_{Y}^{I}=a_{y 0} T \\
& \Delta H_{Z}^{I}=\frac{T}{2}\left(a_{z 1}+b_{x 1}\right)
\end{aligned},
$$

Supposing the wheel unloading is conducted after every $N$ orbital circles, the needed angular momentums of the reaction wheels can be written as

$$
\begin{aligned}
& H_{X \text { wheelneed }}=\Delta H_{X}^{I} \cdot N \\
& H_{Y \text { wheelneed }}=\Delta H_{Y}^{I} \cdot N, \\
& H_{Z \text { wheelneed }}=\Delta H_{Z}^{I} \cdot N
\end{aligned}
$$

\begin{tabular}{|c|c|c|c|c|c|c|}
\hline Classifications & Locations & Designations & $\begin{array}{l}\text { Lower } \\
\text { bounds }\end{array}$ & $\begin{array}{l}\text { Upper } \\
\text { bounds }\end{array}$ & $\begin{array}{l}\text { Initial } \\
\text { values }\end{array}$ & $\begin{array}{l}\text { Optimization } \\
\text { results }\end{array}$ \\
\hline \multirow[t]{7}{*}{ Honeycomb core thickness (m) } & Top panel in payload cabin & PLTT & 0.035 & 0.05 & 0.045 & 10.035 \\
\hline & Base panel in payload cabin & PLBT & 0.035 & 0.05 & 0.045 & 0.035 \\
\hline & Top panel in platform & PFTT & 0.03 & 0.045 & 0.04 & 0.040302 \\
\hline & Middle panel in platform & PFMT & 0.015 & 0.03 & 0.02 & 0.015 \\
\hline & Base panel in platform & PFBT & 0.035 & 0.05 & 0.045 & 0.035 \\
\hline & Side panels & SCT & 0.015 & 0.03 & 0.02 & 0.015 \\
\hline & Payload cabin clapboards & PLRT & 0.01 & 0.25 & 0.02 & 0.01 \\
\hline Panel thickness (m) & Joint ring & $\mathrm{AT}$ & 0.015 & 0.06 & 0.05 & 0.015 \\
\hline \multirow[t]{16}{*}{ Beam sections Width/Depth(m) } & Top circular beams width & TTRW & 0.015 & 0.04 & 0.03 & 0.015 \\
\hline & Top circular beams depth & TTRH & 0.015 & 0.04 & 0.02 & 0.015 \\
\hline & Middle circular beams width & TMRW & 0.015 & 0.04 & 0.03 & 0.015 \\
\hline & Middle circular beams depth & TMRH & 0.015 & 0.04 & 0.02 & 0.015 \\
\hline & Base circular beams width & TBRW & 0.015 & 0.04 & 0.03 & 0.015 \\
\hline & Base circular beams depth & TBRH & 0.015 & 0.04 & 0.02 & 0.015 \\
\hline & Vertical beams width & TVW & 0.015 & 0.04 & 0.03 & 0.015 \\
\hline & Vertical beams depth & TVH & 0.015 & 0.04 & 0.02 & 0.015 \\
\hline & Horizontal beams width & THW & 0.015 & 0.04 & 0.03 & 0.015 \\
\hline & Horizontal beams depth & THH & 0.015 & 0.04 & 0.02 & 0.015 \\
\hline & Tilted beams width & TAW & 0.015 & 0.04 & 0.03 & 0.031212 \\
\hline & Tilted beams depth & TAH & 0.015 & 0.04 & 0.02 & 0.024201 \\
\hline & Top crossed beams width & PFTW & 0.015 & 0.04 & 0.03 & 0.015 \\
\hline & Top crossed beams depth & PFTH & 0.015 & 0.04 & 0.02 & 0.015 \\
\hline & Base crossed beams width & PFBW & 0.015 & 0.04 & 0.03 & 0.015 \\
\hline & Base crossed beams depth & PFBH & 0.015 & 0.04 & 0.02 & 0.015 \\
\hline
\end{tabular}

The estimate models of wheel mass and power are given as below

$$
\begin{aligned}
m_{\text {wheel }} & =\left\{\begin{array}{l}
2.7+\frac{18}{55}\left(H_{\text {wheel }}-0.5\right) 0.5 \leq H_{\text {wheel }}<6 \\
5.8+\frac{17}{90}\left(H_{\text {wheel }}-6\right) 6 \leq H_{\text {wheel }} \leq 15
\end{array},\right. \\
P_{\text {wheel }} & =\left\{\begin{array}{l}
80.5 \leq H_{\text {wheel }}<6 \\
116 \leq H_{\text {wheel }} \leq 15
\end{array},\right.
\end{aligned}
$$

Table 9 Optimization results of structural design variables 
Table 10 Optimization results of constraints

\begin{tabular}{llllll}
\hline Classifications & Designations & Lower bounds & Upper bounds & Initial values & Optimization results \\
\hline System & $m_{p}$ & - & 600 & 575.40 & 580.57 \\
& $m_{\text {total }}$ & - & 1250 & 1278.50 & 1250.40 \\
& $\Delta v-\Delta v_{\text {need }}$ & 0 & - & 18.37 & 187.89 \\
Attitude control & $g_{G N C}$ & 0 & - & 0.97 & $2.73 \mathrm{E}-04$ \\
Power & $P_{\text {saEOL }}-(1+5 \%) P_{S C}$ & 0 & - & 86.61205 & $1 \mathrm{E}-04$ \\
& $D O D_{A v g}$ & - & $30 \%$ & $25.80 \%$ & $30.00 \%$ \\
Structure & $D O D_{\max }$ & - & $35 \%$ & $28.08 \%$ & $33.18 \%$ \\
\hline
\end{tabular}

For other mass and power of control subsystem, including sensors, onboard computer, other mechanical parts, etc., are considered as constant in the work, which are total $70.4 \mathrm{~kg}$ and $70.8 \mathrm{~W}$ respectively.

\subsubsection{Analysis example of disturbance moment wheel parameters}

For examples, a sun-synchronous satellite is given with conditions as in Table 4, and the combined effect of practical disturbance moments and their approximation are shown in Fig. 8.

If the reaction wheel unloading is conducted every 14 orbital circles ( 1 day), according to the values of angular momentums $\Delta H_{i}^{I}$ in Table 5 which are then multiplied by 14 , each wheel should select the product type possessing more than $12 \mathrm{Nms}$ angular momentums.

\subsection{Analysis modeling for power disciplinary}

In power disciplinary, the analysis models are established to compute responding functions $P_{S a E O L}$ and $P_{S C}$, which are the solar array power in the end of lifetime and the required power of satellite, as well as $D O D_{A v g}$ and $D O D_{\max }$, which are the battery average and maximum discharge depths. To establish a more accurate model which takes into the consideration of practical application, the modeling method is completed according to the approaches in (Yan 2001).

\subsubsection{Required power of satellite}

The satellite consumed power can be regarded as permanent power $P_{0}$ and short-term power. The permanent power is constantly consumed. However, the short-term power is only consumed when the temporary working device(s) or payload(s) work. There are three working modes A, B and C of the short-term power, i.e. mode A- only thermal control device works during the whole Earth shadow period; mode B- both data transmission and signal test devices work in light period, and the operating durations are 6 minutes and 9 minutes respectively; and mode $\mathrm{C}$ - both thermal control and data transmission devices work in Earth shadow period, and the operating durations are $T_{e}$ and 6 minutes respectively. Considering that the orbital altitude of the designed maneuver satellite is in the range of $400 \sim 1000 \mathrm{~km}$, it will be 14 orbital circles each day. The working modes of shortterm power in these 14 circles are arranged as in Table 6. More details are shown in ESM in Section 3.1.

Table 11 Optimization results of other global parameters

\begin{tabular}{|c|c|c|c|}
\hline Classifications & Designations & Initial values & Optimization results \\
\hline \multirow[t]{3}{*}{ System } & Moment of inertia $\left(\mathrm{kgm}^{2}\right) \quad I_{x x}$ & 444.99 & 431.30 \\
\hline & Moment of inertia $\left(\mathrm{kgm}^{2}\right) \quad I_{y y}$ & 534.15 & 516.30 \\
\hline & Moment of inertia $\left(\mathrm{kgm}^{2}\right) \quad I_{z z}$ & 549.75 & 530.56 \\
\hline \multirow[t]{2}{*}{ Attitude control } & Subsystem mass (kg) & 85.78 & 84.52 \\
\hline & Subsystem power (W) & 102.80 & 102.80 \\
\hline \multirow[t]{5}{*}{ Power } & Initial power output(W) & 805.02 & 737.37 \\
\hline & Ending power output(W) & 753.05 & 689.76 \\
\hline & Mass of solar array (kg) & 15.60 & 14.27 \\
\hline & Mass of battery (kg) & 25.51 & 22.27 \\
\hline & Subsystem mass (kg) & 50.81 & 46.24 \\
\hline Structure & Structural mass $(\mathrm{kg})$ & 216.47 & 189.02 \\
\hline
\end{tabular}




\subsubsection{Calculation of responding functions}

(1) Lower bound of solar array output power

According to the required power of the satellite and the given parameters such as loss and efficiency factors, considering energy balance, we can calculate the needed remained power $P_{c}$ and the electrical margin of battery in every revolution $Q_{D F i}(i=1, \cdots, n)(n=14$ circles in this study) (see the ESM of Section 3.2 for details), and then the lower bound of the solar array output power $P_{S C}$ can be determined as below

$P_{S C}=P_{0}+P_{c}$.

(2) Solar array power in end of lifetime

In end of lifetime, the general output power of solar array $P_{E O L}$ is shown as

$P_{E O L}=P_{B O L} \cdot F_{R A D} \cdot F_{U V} \cdot F$

where $P_{B O L}$ is the output power of solar array in early days (see ESM of Section 3.2 for detail) (Ma 2001), $F_{R A D}$, $F_{U V}$ and $F$ are decay factors for particle-radiation, ultraviolet radiation and other elements. Finally the solar array power in end of lifetime $P_{S a E O L}$ in optimization problem (14) can be obtained when setting the worst condition in which the sun-angle $\eta$ is usually taken as the maximum, i.e. $P_{S a E O L}=\min P_{E O L}$.

(3) Battery maximum and average discharging depths

The depth of discharge (designated DOD) for the battery group is defined as the ratio of its discharged capacitance $C$ to its rated capacitance $C_{s}$, i.e. $D O D=C / C_{s}$, which can be further presented as (Ma 2001)

$D O D=\frac{Q_{F}}{V_{D B} \cdot F_{1} \cdot C_{S}}$,

where $F_{1}$ is a constant related to discharging efficiency, taken $F_{1}=0.9$ in this work; and

$V_{D B}=\left(N_{b a t}-N_{w b}\right) \cdot V_{d l}-V_{d i o}-V_{l i n e}$,

where $V_{d l}$ is the average discharging voltage of a single battery, for instance taken $1.25 \mathrm{~V}$ here; $V_{\text {dio }}$ and $V_{\text {line }}$ are the voltage drops of isolating diode and power supply cable; $N_{b a t}$ and $N_{w b}$ are the numbers of series-wound and warm backup single batteries in battery group respectively, which are usually taken as 18 and 1 or $2 ; Q_{F}$ is the discharged energy of battery group in an orbital circle period, which can be calculated by using the formula as below

$Q_{F}=Q_{C 0}+Q_{C 1}$. where $Q_{C 0}$ and $Q_{C 1}$ are charged energy and additional charged energy of battery group, presented in the ESM of Section 3.2. $Q_{F}$ also changes vs. different orbit circles due to working mode changes, hereby using $Q_{F i}(i=1, \cdots, n)$ to present $Q_{F}$ in the $i$-th orbit. The battery maximum and average discharging depths, $D O D_{\max }$ and $D O D_{\text {avg }}$, can be obtained when the battery discharged energy $Q_{F}$ in Eq. (40) takes the maximum and average in $n$ orbit circles, i.e. $\left(Q_{F}\right)_{\max }=\max \left(Q_{F i} i=1, \cdots, n\right)$ and $\left(Q_{F}\right)_{\text {avg }}=\sum_{i=1}^{n} Q_{F i} / n$.

(4) Mass of power subsystem

The mass of solar array is expressed as

$m_{s a}=\rho_{s a} \cdot A_{s a}$,

where $\rho_{s a}$ is the areal density that ranges from $2.3(\mathrm{Si}) \sim 2.6(\mathrm{GaAs} / \mathrm{Ge}) \mathrm{kg} / \mathrm{m}^{2}$.

The mass of battery group is shown as

$m_{b a t}=C_{S} \cdot V_{D B} / \gamma_{b}$,

where $\gamma_{b}$ is the specific energy, for example $\gamma_{b}=$ $39.6 \mathrm{Wh} / \mathrm{kg}$ if $\mathrm{H}_{2}$-Ni battery is chosen as in this work.

Considering the mass of power controller $m_{c}$ that was taken as constant $9.7 \mathrm{~kg}$ in present work, the mass of power system can be finally described as

$m_{\text {power }}=m_{s a}+m_{\text {bat }}+m_{c}$.

\subsection{Analysis modeling for structural disciplinary}

The structural subsystem is established on the basis of a given satellite configuration, and a finite element model is adopted to conduct structural analysis. As an example in structural disciplinary of this work, structural optimization is executed on the main load-bearing parts and all beams in the frame structure possess rectangular hollow cross-sections. Structure subsystem analysis is completed to obtain the subsystem mass, the natural frequencies, which are the local constraints, and the moment of inertia around the three main axes, which are the coupled input of the control subsystem analysis.

The satellite body is an octahedron, with $1600 \mathrm{~mm}$ height and $1800 \mathrm{~mm}$ as the diameter of the octagonal inscribed circle, consisting of a payload module, a main structure platform, a transition module between them, and a $\Phi 1194 \mathrm{~mm}$ joint ring at the bottom. The main load-bearing beam frame is placed in the main structure platform with four fuel tanks inside. See Fig. 9 for the structural finite element (FE) model, with some shell elements erased. The mass of other 
subsystems is modeled in the FE model by nonstructural mass (NSM) or lumped mass linked to the structure according to layout of the devices. The values of the NSM and lumped mass are parameterized in the FE model. Designvariables in the structure subsystem are the heights of the honeycomb core and cross-sections of the box beams with thickness value of $1.5 \mathrm{~mm}$ initially. The bounds on all box beam sections of height and width are set between 15 40 $\mathrm{mm}$ shown in Table 9. See the ESM in Section 4 for more details, like structural materials and their properties.

\section{Optimization results}

First of all, the initial values of parameters in this maneuver satellite were set on the basis of the orbital altitude of $850 \mathrm{~km}$. And from the disciplinary constraints, the initial design for every disciplinary could be considered as an optimal system in practical engineering design. The system optimization problem with corresponding design variables and constraints was then established, and the optimization results were obtained with NNCO method.

\subsection{Optimization results of objectives}

The optimization results of the system level objectives and corresponding iteration history are shown in Table 7 and Fig. 10, respectively. From Table 7, it could be seen that compared with the initial design, the velocity increment and the total mass reach their corresponding balance in multidisciplinary and a relatively compatible state has been arrived at. And the total mass of satellite basically satisfies the constraint.

The iteration history of the compatibility constraints are demonstrated in Fig. 11. We can see that the compatibility constraints meet the constraint conditions when the optimization iteration terminates. So it could be said that this system with the optimization design is compatible. During the optimization, the normalization factors for the velocity increment and total mass, i.e. $\Delta v_{\text {scale }}$ and $m_{\text {total }}^{\text {scale }}$, are $50 \mathrm{~m} / \mathrm{s}$ and $1200 \mathrm{~kg}$, respectively, the weighted coefficient $W$ is 1000 , the initial value of the penalty parameter $\gamma$ is 1 , and its update expression is $\gamma_{k+1}=5 \gamma_{k}$.

\subsection{Optimization results of design variables}

The optimal results of system design variables and structural size dimension design variables are listed in Tables 8 and 9, respectively. In Table 8 , the value of orbital height changes most compared with other design variables, which illustrates that the objective is more sensitive to the orbital height. From the results in Table 9, it can be seen that many optimal dimensions reach to corresponding lower bounds.

\subsection{Optimizations results of constraints}

Table 10 shows the optimization results of the constraints. As for the meanings of the designations, see Table 3. Obviously the initial design doesn't satisfy the total mass constraint, and after optimization, the total mass almost arrives at the constraint bound. Some other parameters, including residue of angular momentum, power residue and battery average discharge depth, have also reached about their corresponding constraint bounds. The 1 st order frequency for the whole satellite is $22 \mathrm{~Hz}$ after optimization, which also meets the given constraint, and the corresponding mode indicates the whole satellite rather than local structures. In addition, the residue of velocity increment changes a lot after optimization. The battery average discharge depth reaches the upper bound, while the battery maximum discharge depth doesn't, which shows that the main factor in battery design lies in the battery average discharge depth.

\subsection{Optimization results of other global parameters}

Table 11 lists the optimal results of other global parameters. Masses of subsystems like attitude control and power haven't changed much after optimization. In the case of small changes in other design variables, parameters of the overall system are relatively stable.

\section{Conclusions}

A multidisciplinary design optimization (MDO) application for a satellite with maneuver capability was investigated. With the velocity increment $\Delta v$ taken as the measurement of maneuvering capability of the studied satellite, the subsystems of attitude control, power, and structures were also established in the MDO model under the collaborative optimization method architecture. By adopting a non-nested $\mathrm{CO}$ method with the software iSIGHT, a program system to solve this problem was developed. The critical factors to address the MDO problem lie in the determination of global parameters and the establishment of optimization and analysis models, which need to reflect the practical application. After optimization, a more compatible and better design is obtained and the integrated performance is improved to some extent. The results also show that the MDO is feasible and effective to cope with the engineering design issues, which is expected to further apply in more practical engineering satellite system designs. 


\section{References}

Alexandrov NM, Lewis RM (2002) Analytical and computational aspects of collaborative optimization for multidisciplinary design. AIAA J 40(2):301-309

Allison J, Walsh D, Kokkolaras M, Papalambros PY, Cartmell M (2006) Analytical target cascading in aircraft design. In: Proceedings of the 44th AIAA aerospace sciences meeting and exhibit, AIAA-2006-1326, pp 9-12

Altair (2009) Altair Hyperstudy. Version 10.0. http://www. altairhyperworks.de/HWTemp1Product.aspx?product_id=10\& AspxAutoDetectCookieAspx.

Blouin VY, Summers JD, Fadel GM, Gu J (2004) Intrinsic analysis of decomposition and coordination strategies for complex design problems. In: Collection of Technical Papers-10th AIAA/ISSMO Multidisciplinary Analysis and Optimization Conference, pp 1853-1864

Braun RD (1996) Collaborative optimization: an architecture for large-scale distributed design. Doctoral Dissertation, Stranford University, California

Braun RD, Moore AA, Kroo IM (1997) Collaborative approach to launch vehicle design. J Spacecr Rocket 34(4):478-486

Brown NF, Olds JR (2006) Evaluation of multidisciplinary optimization techniques applied to a reusable launch vehicle. J Spacecr Rocket 43(6): 1289-1300

Chen S, Huang H (2010) Optimum design of a space frame and its application in satellite structure. J Spacecr Rocket 47(6):10631066

Choi YC, Noh K-H, Lee J-W, Byun Y-H, Yu YH (2006) Mission and system design of air-Launching rocket using multidisciplinary optimization approach. In: 47th AIAA/ASME/ASCE/AHS/ASC Structures, Structural Dynamics, and Materials Conference, Newport, Rhode Island

Cramer EJ, Dennis JE Jr., Frank PD, Lewis RM, Shubin GR (1994) Problem formulation for multidisciplinary optimization. SIAM J Optim 4(4):754-776

EngineousSoftware (2004) iSIGHT Version 8.0. http://www. engineous.com/product_iSIGHT.htm.

Esteco (2011) ModeFrontier - explore new frontiers of innovation. Esteco. http://www.esteco.com/home.html.

Ilan K, Steve A, Robert B, Peter G, Ian S (1994) Multidisciplinary optimization methods for aircraft preliminary design. In: 5th AIAA/USAF/NASA/ISSMO Symposium on Multidisciplinary Analysis and Optimization, Panama City Beach

Jaroslaw S, Jeremy SA, Robert R, Sandusky J (1998) Bi-level integrated system synthesis (BLISS). AIAA J 38(1):164-172
Ma S (2001) Satellite power technology. China Astronautical Publishing House, Beijing

Nobuhiro Y, Shinji S, Takeshi T, Hideyuki T, Takeshi K (2005) Multidisciplinary design optimization of SSTO space plane considering rigid body characteristics. Paper presented at the 43rd AIAA Aerospace Sciences Meeting and Exhibit, Reno, Nevada

PhoenixIntegration (2008) ModelCenter 8.0 Help. http://www. phoenix-int.com/products/index.html.

Renaud J, Gabriele G (1993) Improved coordination in nonhierarchic system optimization. AIAA J 31(12):2367-2373

Sobieszczanski-Sobieski J (1982) A linear decomposition method for large optimization problems. Blueprint for development. NASA TM-83248

Sobieszczanski-Sobieski J (1989) Optimization by decomposition: a step from hierarchic to non-hierarchic systems. NASA STI/Recon Technical Report N 89:25149

Sobieszczanski-Sobieski J (1990) Sensitivity analysis and multidisciplinary optimization for aircraftdesign-Recent advances and results. J Aircr 27(12):993-1001

Sobieszczanski-Sobieski J, Chopra I (1990) Multidisciplinary optimization of aeronautical systems. J Aircr 27(12):977-978

Sobieszczanski-Sobieski J, Haftka RT (1997) Multidisciplinary aerospace design optimization: survey of recent developments. Struct Optim 14(1):1-3

Sobieszczanski-Sobieski J, James BB, Dovi AR (1985) Structural optimization by multilevel decomposition. AIAA J 23(11):17751782

Tan C, Pang B, Zhang L, Huang H (2008) Multidisciplinary optimization in earth observation satellite main parameters. J Beijing Univ Aeronaut Astronaut 34(5):529-532

Wertz JR, Larson WJ (1992) Space mission analysis and design. Microcosm Press, California

Wu B, Huang H, Wu W (2012) A nonnested collaborative optimization method for multidisciplinary design problems. In: 2012 IEEE 16th International Conference on Computer Supported Cooperative Work in Design (CSCWD). IEEE, pp 148-152

Xiao Y (1995) Spacecraft dynamic theory. China Astronautical Publishing House, Beijing

Xu F (2002) Satellite engineering. China Astronautical Publishing House, Beijing

Yan W (2001) Research of Sun synchronization orbit satellite power system design computation method. Chin Space Sci Technol 21(2):19-25

Yokoyama N, Suzuki S, Tsuchiya T, Taguchi H, Kanda T (2007) Multidisciplinary design optimization of space plane considering rigid body characteristics. J Spacecr Rocket 44(1):121131 